\title{
Search for electric dipole moments at storage rings
}

\author{
C. J. G. Onderwater
}

Published online: 28 February 2012

(C) The Author(s) 2012. This article is published with open access at SpringerLink.com

\begin{abstract}
Permanent electric dipole moments (EDMs) violate parity and timereversal symmetry. Within the Standard Model (SM) they are many orders of magnitude below present experimental sensitivity. Many extensions of the SM predict much larger EDMs, which are therefore an excellent probe for the existence of "new physics". Until recently it was believed that only electrically neutral systems could be used for sensitive searches of EDMs. With the introduction of a novel experimental method, high precision for charged systems will be within reach as well. The features of this method and its possibilities are discussed.
\end{abstract}

Keywords Permanent electric dipole moment • Standard model test • New physics search

\section{Motivation}

The symmetry properties of fundamental processes and particles are a strong guide to understand the underlying interactions. The QED Lagrangian in the current Standard Model (SM) predicts that all electromagnetic observables are even under the discrete symmetries $\mathcal{C}$ (charge conjugation), $\mathcal{P}$ (parity) and $\mathcal{T}$ (time reversal) individually and thus under each combinations of them. Strong interaction observables, described by QCD, are also predicted to be even under $\mathcal{C}, \mathcal{P}$ and $\mathcal{T}$, with the exception of those proportional to $\bar{\theta}$ which are $\mathcal{P}$ and $\mathcal{T}$-odd [1]. The weak interaction violates both $\mathcal{P}$ and $\mathcal{C}$ because of the handedness of the coupling of the $\mathrm{W}$ and Z-bosons. Many observables are even under their combination $\mathcal{C} \mathcal{P}$. Nevertheless, the weak interaction also predicts $\mathcal{C} \mathcal{P}$-odd ones. These are all proportional to the Jarlskog invariant, $J \propto \sin ^{2} \theta_{12} \sin \theta_{23} \sin \theta_{13} \sin \delta \sim 3 \times 10^{-5}$ [2]. Here $\theta_{12}, \theta_{23}$ and $\theta_{13}$ are the quark flavor mixing angles and $\delta$ the $\mathcal{C P}$-violating complex phase associated

C. J. G. Onderwater $(\bowtie)$

KVI, University of Groningen, Zernikelaan 25, 9747AA Groningen, The Netherlands e-mail: onderwater@kvi.nl 
with the Kobayashi-Maskawa mechanism [3]. The SM is built on the assumption of Lorentz invariance and hence the invariance of the combination $\mathcal{C P} \mathcal{T}$. Consequently $\mathcal{T}$ and $\mathcal{C P}$ violation are equivalent.

In the $\mathrm{SM}$ there are thus two sources of $\mathcal{C P} / \mathcal{T}$ violation, $\bar{\theta}_{Q C D}$ and $J$. The magnitude of the former is as-of-yet undetermined, $|\bar{\theta}|<\mathcal{O}\left(10^{-11}\right)$, whereas the smallness of the latter guarantees that $\mathrm{SM} \mathcal{C} \mathcal{P}$-odd observables are generally small.

Violation of $\mathcal{C P}$ is also expected to be necessary to explain the baryon asymmetry in the universe [4]. The baryon asymmetry predicted from the SM and the Cosmological Standard Model falls short of the observed one by as much as ten order of magnitude. This suggests the presence of additional sources of $\mathcal{C P}$-violation beyond those incorporated in the SM.

Permanent electric dipole moments (EDMs) are an excellent tool to search for such additional sources of $\mathcal{C} \mathcal{P}$-violation [5]. EDMs break both $\mathcal{P}$ and $\mathcal{T}$, which is manifest when considering the field dependent part of the interaction Hamiltonian $\mathcal{H}$ for a particle in an electric field $\mathbf{E}$ and a magnetic field $\mathbf{B}$,

$$
\mathcal{H}=-(\boldsymbol{\mu} \cdot \mathbf{B}+\mathbf{d} \cdot \mathbf{E})=-(\mu \mathbf{B}+d \mathbf{E}) \cdot \frac{\mathbf{J}}{J} .
$$

The second equality holds because the spin $\mathbf{J}$ is the only vector in the rest frame of a fundamental particle. The electric and magnetic dipole moments must point along it; $\mu=g(e / 2 m)$ and $d=\eta(e / 4 m)$ are the respective proportionality constants (using $\hbar=c=1$ ). In units of the Bohr magneton $e / 2 m$ they are respectively given by the dimensionless moments $g$ and $\eta / 2$. When $d \neq 0$ this Hamiltonian violates both $\mathcal{P}$ and $\mathcal{T}$.

Particles acquire non-zero EDMs through $\mathcal{C P}$ violating radiative corrections. Quark mixing, i.e. $\delta$, contributes only through three or more weak-interaction loop corrections. This makes these EDMs extremely small, of order $10^{-31} e \cdot \mathrm{cm}$ for hadronic systems down to $10^{-41} e \cdot \mathrm{cm}$ for leptons. This is far below present detection limits [6]. Hadronic systems may also acquire an EDM through $\bar{\theta}$ without the need for multiple loops. The non-observation of the neutron EDM limits the magnitude of $|\bar{\theta}|<\mathcal{O}\left(10^{-11}\right)$.

In many proposed extensions of the SM, the need for multiple loops is not present, and EDMs may occur even at first order [7]. For example, many supersymmetric (SUSY) models predict a neutron EDM

$$
d_{n}(S U S Y) \sim \sin \delta_{C P}^{S U S Y}\left(\frac{1 \mathrm{TeV}}{M_{S U S Y}}\right)^{2} \times 10^{-25} e \cdot \mathrm{cm} .
$$

Once the SUSY mass scale $M_{S U S Y}$ is determined, the $\mathcal{C P}$ violating phase $\delta_{C P}^{S U S Y}$ can be determined using EDMs.

The first observation of a non-zero EDM would already unambiguously establish the presence of physics beyond the SM. Different forms of new physics manifest themselves differently already at the level of the fundamental fermions and their interactions, and propagate into increasingly larger composite systems, from hadrons to nuclei, atoms and molecules [8]. At each stage the appropriate theory needs to be applied. The most stringent limits on quark and proton EDM, as well as on $\mathcal{C P}$ violating electron-nucleus interactions are derived from the EDM search on the ${ }^{199} \mathrm{Hg}$ atom [9]. The most strict electron EDM limit stems from the YbF molecule [10]. The muon EDM is the only fundamental particle for which the EDM was obtained directly [11]. 
A single EDM measurement cannot be traced back to a specific source of $\mathcal{C P}$ violation. Several complementary measurements are necessary. For example, the combination of the neutron EDM combined with that for the proton, deuteron and possibly helion and triton makes it possible to distinguish new sources of $\mathcal{C P}$ violation from that introduced by $\bar{\theta}$ [12]. The uncertainties in the theory to describe light nuclei are well under control permitting reliable predictions [13-15]. Light nuclei thus offer the theoretically cleanest way to study hadronic $\mathcal{C P}$ violation.

Light nuclei cannot be probed for an EDM using atom- or molecule-based methods because of shielding effects [16]. Several experimental methods that circumvent these problems make use of the motional electric field a fast moving particle experiences when traversing a magnetic field [17-20]. These methods provide direct access to the very interesting realm of light nuclei, which so far have not been examined for EDMs. Also the muon can be probed sensitively, offering the unique possibility to explore the flavor structure of fundamental particles.

\section{Storage ring techniques}

A charged particle with magnetic moment anomaly $a$ and EDM $\eta$ moving in an electromagnetic field will exhibit spin precession. The evolution of the spin $\mathbf{S}$ is described by the (simplified) BMT equation [21],

$$
\frac{d \mathbf{S}}{d t}=\frac{e}{m} \mathbf{S} \times\left[a \mathbf{B}+\left(\frac{1}{\gamma^{2}-1}-a\right) \boldsymbol{\beta} \times \mathbf{E}+\frac{\eta}{2}(\mathbf{E}+\boldsymbol{\beta} \times \mathbf{B})\right] \equiv \mathbf{S} \times \boldsymbol{\Omega} .
$$

The first two terms arise from the interaction of the magnetic moment with the magnetic field, whereas the last term is due to the interaction of the EDM with the electric field. The EDM interacts with the electric field in the rest-frame of the particle, which may have a strength $E^{C M} \sim E+v B \sim \mathrm{GV} / \mathrm{m}$ far in excess of those attainable in the laboratory.

In a purely magnetic storage ring the spin precesses about $\boldsymbol{\Omega}$ which is tilted with respect to $\mathbf{B}$ by an angle $\psi \simeq \eta \beta / 2 a$. The precession rate increases to $\Omega=\sqrt{1+\psi^{2}} \Omega_{0}$ with $\Omega_{0}=a(e / m) B$. This quadratic sensitivity of the precession rate precludes a sensitive measurement of $\eta$. Because $\boldsymbol{\Omega}$ is tilted with respect to $\mathbf{B}$ the spin component along the magnetic field oscillates with an amplitude that depends linearly on the EDM. In the muon g-2 experiment at BNL this was used to limit the muon EDM [11]. It will be used again in the new muon g-2 experiment at Fermilab [22]. The muon EDM limit of $10^{-19} e \cdot \mathrm{cm}$ corresponds to a tilt in the precession plane of order 1 microradian.

The statistical power of an EDM experiment is proportional to $P E \sqrt{N} T A$ with polarization $P$, effective electric field $E$, number of particles $N$, characteristic time scale $T$ and analyzing power $A$. The sensitivity of the $\mathbf{B}$-only method is limited by the short precession cycle, which determines $T$.

Reducing the precession rate will prolong $T$. This can be done by applying a suitably chosen combination of radially oriented electric and vertically oriented magnetic fields. For

$$
\frac{E}{B}=\frac{a \beta}{1-(1+a) \beta^{2}}
$$


the first two terms in (3) cancel, so that $\boldsymbol{\Omega}=\eta / 2(e / m)(\mathbf{E}+\boldsymbol{\beta} \times \mathbf{B})$. The spin precession rate is now entirely determined by the EDM. The spin precesses about the electric field in the rest frame of the particle, which is oriented radially in the laboratory frame. The signature of an EDM is the change of the polarization component out of the particle orbit plane, generally along the magnetic field.

For particles with positive $a$ a "magic" momentum $p_{\text {magic }}=\frac{m}{\sqrt{a}}$ exists. At this momentum the particles can be stored in an all-electric setup with $B=0$. The bending radius $\rho$ of a particle with mass $m$ moving in an electric field $E$ is

$$
\rho=\frac{1}{\sqrt{a(a+1)}} \frac{m}{E} .
$$

For a proton $p_{\text {magic }} \simeq 700 \mathrm{MeV} / c$ and $\rho \simeq 42 \mathrm{~m}$, assuming $E=10 \mathrm{MV} / \mathrm{m}$. The spin precesses at a rate of $\Omega / d=2 E \simeq 10^{20} \mathrm{rad} / \mathrm{s} /(e \cdot \mathrm{cm})$. At the expected sensitivity for $\Omega$ of $1 \mathrm{nrad} / \mathrm{s}$ a proton EDM of $d_{p}=10^{-29} e \cdot \mathrm{cm}$ can be measured. Efforts are ongoing to realize such an experiment [23].

An all-electric setup is not feasible for particles with small $a$ and impossible for those with a negative one. A combination of electric and magnetic fields is necessary to "freeze" the spin. Expressed in $E$ the EDM-induced spin precession rate and corresponding bending radius are given by

$$
\Omega=2 d(E+v B)=\frac{a+1}{a \gamma^{2}} 2 d E \quad \text { and } \quad \rho=\frac{a \beta^{2} \gamma^{3}}{a+1} \frac{m}{E}
$$

Both from the point of view of the spin precession rate and the size of the setup low momenta are preferred. The electric field is effectively amplified by $(a+1) / a \gamma^{2}$. This becomes sizable for particles with a small $a$ (see [24]). For the electron or muon with $a \simeq 0.00116$ this is $(a+1) / a \simeq 860$.

For muons with $p=500 \mathrm{MeV} / c$ and $E=2.2 \mathrm{MV} / \mathrm{m}$ as proposed in [25] $\rho=7 \mathrm{~m}$. A considerably smaller setup with $\rho=0.42 \mathrm{~m}$ is proposed in [26] with $p=125 \mathrm{MeV} / c$ and $E=0.64 \mathrm{MV} / \mathrm{m}$. The projected sensitivities are about the same at $d_{\mu} \simeq 10^{-16} e$. $\mathrm{cm} / \sqrt{N}$, with $N$ the number of detected muon decays. At existing muon facilities $N=10^{12}$ could be collected yielding a statistics limited sensitivity of $d_{\mu} \simeq 10^{-22} e$. $\mathrm{cm}$. This improves the current limit by three orders of magnitude. This experiment could well serve as a small-scale low-cost demonstration of this novel technique. At a future high-intensity muon facility this can be further improved by several orders of magnitude.

At the Forschungszentrum Jülich the possibilities for a light-ion EDM facility are explored [27]. Several options for an "all-in-one" storage ring were presented to search for EDMs on protons, deuterons and ${ }^{3} \mathrm{He}$ [28]. For a $p_{p}=435 \mathrm{MeV} / c$, $p_{d}=1000 \mathrm{MeV} / c$ and $p^{{ }^{3} \mathrm{He}}=765 \mathrm{MeV} / c$ a single ring with a bending radius of $10 \mathrm{~m}$ can be constructed requiring $B<0.5 \mathrm{~T}$ and $E<17 \mathrm{MV} / \mathrm{m}$.

\section{Outlook}

A sensitive EDM search using a storage ring requires besides statistical also systematic precision. Current R\&D efforts address several aspects that affect both. A system is being developed to reliably generate the electric field strengths of $10 \mathrm{MV} / \mathrm{m}$ planned for the proton EDM search. Such systems have been employed 
on a much smaller scale as electrostatic separators at e.g. the AGS at BNL and the Tevatron at FNAL. A critical aspect is the alignment of the electric field with respect to the magnetic field. Spin and beam dynamics must be understood at an unprecedent level of precision to guarantee optimal statistical precision through a long spin coherence time and to exclude or reduce systematic uncertainties to an acceptable level. An active research program is underway using the COSY facility at the Forschungszentrum Jülich. A lower limit on the spin coherence time of $75 \mathrm{~s}$ was demonstrated already, just one order of magnitude below the goal for the proton and deuteron EDM experiments [29]. Also at COSY a scheme to efficiently measure deuteron polarization and to correct for systematic errors was demonstrated [30]. The demonstrated sensitivity is sufficient to reach the proposed sensitivity of $d_{d}=10^{-29} e \cdot \mathrm{cm}$.

In conclusion storage rings make it possible to enter new territory in the search for EDMs. It is expected that an experiment on the proton and deuteron can be realized in the near future. A first small-scale implementation of a storage ring could be realized already now to search for a muon EDM. They make it possible to directly probe charged particles with competitive sensitivity. Such systems have a complementary sensitivity to new sources of CP-violation and may help to pin-down the last unconfirmed source of CP-violation in the Standard Model, $\bar{\theta}$.

Acknowledgements The author is indebted to his colleagues at KVI, PSI, RCNP and the storage ring-EDM collaboration for valuable discussions. The research described in this paper was partly financed by an Innovative Research grant (\#680-47-203) from the Dutch Organization for Fundamental Research (NWO).

Open Access This article is distributed under the terms of the Creative Commons Attribution License which permits any use, distribution, and reproduction in any medium, provided the original author(s) and the source are credited.

\section{References}

1. Cheng, H.Y.: The strong CP problem revisited. Phys. Rep. 158, 1 (1988)

2. Jarlskog, C.: A basis independent formulation of the connection between quark mass matrices, CP violation and experiment. Phys. Z. C29, 491 (1985)

3. Kobayashi, M., Maskawa, T.: CP violation in the renormalizable theory of weak interaction. Prog. Theor. Phys. 49, 652 (1973)

4. Sakharov, A.D.: Expanding universe and the appearance of a nonuniform distribution of matter. Pis'ma Z. Eksp. Teor. Fiz. 5, 32 (1967) [JETP Lett. 5, 24 (1967)]

5. Pospelov, M., Ritz, A.: Electric dipole moments as probes of new physics. Ann. Phys. 318, 119 (2005)

6. Khriplovich, I.B., Lamoreaux, S.K.: CP Violation Without Strangeness. Springer (1997)

7. Sandars, P.G.H.: Electric dipole moments of charged particles. Contemp. Phys. 42, 97 (2001)

8. Gould, C.R., Davis, E.D.: Time reversal invariance in nuclear physics: from neutrons to stochastic systems. In: Beyer, M. (ed.) CP Violation in Particle, Nuclear and Astrophysics, p. 207. Springer (2002)

9. Griffith, W.C., et al.: Improved limit on the permanent electric dipole moment of Hg-199. Phys. Rev. Lett. 102, 101601 (2009)

10. Hudson, J.J., et al.: Improved measurement of the shape of the electron. Nature 473, 493 (2011)

11. Bennett, G.W., et al.: An improved limit on the muon electric dipole moment. Phys. Rev. D80, 052008 (2009)

12. de Vries, J., et al.: Electric dipole moments of light nuclei from chiral effective field theory. Phys. Rev. C84, 065501 (2011) 
13. Liu, C.-P., Timmermans, R.G.E.: P- and T-odd two-nucleon interaction and the deuteron electric dipole moment. Phys. Rev. C70, 055501 (2004)

14. Afnan, I.R., Gibson, B.F.: Model dependence of the (2)H electric dipole moment. Phys. Rev. C82, 064002 (2010)

15. de Vries, J., Mereghetti, E., Timmermans, R.G.E., van Kolck, U.: P and T violating form factors of the deuteron. Phys. Rev. Lett. 107, 091804 (2011)

16. Schiff, L.I.: Measurability of nuclear electric dipole moments. Phys. Rev. 132, 2194 (1963)

17. Berley, D., Gidal, G.: Search for an electric dipole moment structure of the muon. Phys. Rev. 118, $1086(1960)$

18. Bailey, J., et al.: New limits on the electric dipole moment of positive and negative muons. J. Phys. G 4, 345 (1978)

19. Farley, F.J.M., et al.: A new method of measuring electric dipole moments in storage rings. Phys. Rev. Lett. 93, 052001 (2004)

20. Orlov, Y.F., Morse, W.M., Semertzidis, Y.K.: Resonance method of electric-dipole-moment measurements in storage rings. Phys. Rev. Lett. 96, 214802 (2006)

21. Bargmann, V., Michel, L., Telegdi, V.L.: Precession of the polarization of particles moving in a homogeneous electromagnetic field. Phys. Rev. Lett. 2, 435 (1959)

22. Carey, R.M., et al.: The new (g-2) experiment: a proposal to measure the muon anomalous magnetic moment to $\pm 0.14 \mathrm{ppm}$ precision. FERMILAB-PROPOSAL-0989

23. Semertzidis, Y.K.: A storage ring proton electric dipole moment experiment: most sensitive experiment to CP-violation beyond the standard model. http://arxiv.org/abs/1110.3378 (2011)

24. Khriplovich, I.B.: Nuclear electric dipole moments at ion storage rings. Hyperfine Interact. 127, 365 (2000)

25. Aoki, M., et al.: J-PARC letter of intent: search for a permanent muon electric dipole moment at the $10^{-24} e \cdot \mathrm{cm}$ level (2003)

26. Adelmann, A., Kirch, K., Onderwater, C.J.G., Schietinger, T.: Compact storage ring to search for the muon electric dipole moment. Phys, J. G37, 085001 (2010)

27. http://www2.fz-juelich.de/ikp/en/future_projects.shtml

28. Lehrach, A.: 485. WE-Heraeus-Seminar. Bad Honnef, Germany. http://www2.fz-juelich.de/ $\mathrm{ikp/edm/en/talks/A.Lehrach.pdf,} \mathrm{4-6} \mathrm{Jul} 2011$

29. Stephenson, E.J., Onderwater, C.J.G., Lenisa, P.: Proc. 8th International Conference on Nuclear Physics at Storage Rings (Stori11) Laboratori Nazionali di Frascati, Italy, 9-14 Oct 2011

30. Brantjes, N.P.M., et al.: Correcting systematic errors in high-sensitivity deuteron polarization measurements. Nucl. Instrum. Methods A664 49, (2012) 